\title{
Computational Study On The Effect Of The Staggered Ribs On Heat Transfer Phenomena Between The Horizontal Plates
}

Ilker Goktepeli $(\mathbb{D})$ Ulas Atmaca (D)

Konya Technical University, Department of Mechanical Engineering, Konya, Turkey

\section{A B S T R ACT}

n terms of multifarious technical applications, various kinds of passive methods are preferred to the active techniques when it comes to increase the amount of convection heat transfer via less energy consumption. As a vortex generator within the scope of the passive method, rib is usually employed to induce the heat transfer enhancement. In this study, the rectangular cross-sectional ribs have been placed to increase the amount of the heat transfer for the staggered arrangement between the horizontal parallel plates. Numerical simulations have been conducted by using $\mathrm{k}-\omega$ SST turbulence model at $\mathrm{Re}=10000$. The rib effect has been comparatively investigated in case of thermal and hydraulic performance presented via the numerical results. Time-averaged results for temperature, pressure, streamwise velocity component and streamline patterns have been presented in terms of contour graphics. Furthermore, heat transfer enhancement by using the ribs has been given depending on the increment ratio of Nusselt numbers. Including friction losses due to the ribs mounted on the plates, the values of thermal performance factor for all ducts have been calculated. According to these results for heat transfer augmentation at $\mathrm{Re}=10000, \mathrm{~h}^{\prime}=0.1$ with $\mathrm{S}^{\prime}=0.5$ having $\eta$ $=1.049$ and $h^{\prime}=0.1$ with $S^{\prime}=0.75$ having $\eta=1.019$ have been recommended rather than the smooth duct.

\section{Keywords:}

Heat transfer enhancement, k- $\omega$ SST, Ribbed plate, Staggered arrangement, Thermal performance

\section{INTRODUCTION}

$\mathrm{H}$ eat transfer enhancement via passive methods is accepted as a popular subject in the engineering disciplines since it draws interests for the power expenditure reduction of the system while improving thermal performance. For instance, mechanism in ribbed channels is a challenging issue for the development of high-performance cooling or heating units requiring less cooling working fluid (Sundén, 2011). However, the working fluid having lower thermal diffusivity is a potential restrictive component for the thermal system in some cases as also pointed out by Kashyap et al. (2018). Therefore, the main goal is to increase the amount of convective heat transfer via various techniques depending on the conditions. It is possible to emphasize that all passive techniques are almost based on the extension of wetted surface area by mounting artificial surface elements or the interruption of both hydrodynamic and thermal boundary layers by escalating the flow mixing. What is more, the interruption of these layers makes compactness higher in the duct (Yang and Chen,
2015). On that note, a rib is not only preferred as an artificial surface element for heat transfer enhancement but also it is used to boost the turbulence intensity for the increment of the thermal performance in the ducts. As stated by Alfarawi et al. (2017), passive heat transfer enhancement method is fundamentally applied for the disruption of the profiles near to the plates as well as the production of recirculation regions in the vicinity of the artificial surface elements. Furthermore, it is known that the vortices tend to follow swirling paths provoking the fluid displacement between the core zones and the plates (Tiggelbeck et al., 1993). Thus, the amount of convection heat transfer is enhanced by dispatching more fresh fluid towards the plates with respect to the study done by Abdollahi and Shams (2015). These aforementioned situations cause the increment of the local heat transfer coefficient. The rectangular cross-sectional ribs have been placed on the plates in this study for this reason. Although various kinds of vortex generators are encountered in the previous works studies done by Ki- 


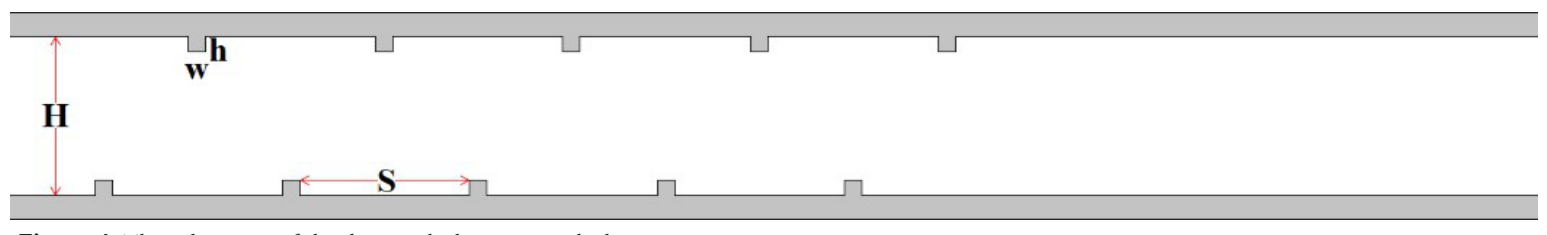

Figure 1. The schematic of the duct with the staggered ribs

licaslan and Sarac (1998), Sripattanapipat and Promvonge (2009), Manca et al. (2011), Wongcharee et al. (2011), Ahmed et al. (2013) and Aslan et al. (2016) on the augmentation of heat transfer, the rib having the rectangular shape is one of the most frequently used elements in terms of heat transfer augmentation. As the rib design is crucial for the level of heat transfer enhancement, also its orientations and arrangements affect the thermal performance for the considered problem. Another supportive study prepared by Moon et al. (2014) reaches a conclusion over the improvement of thermal performance via the ribs. What is more, it has been explained with the influence of the rib shape and its arrangement on the separation bubble and the fluctuations triggering the turbulent kinetic energy. If it is briefly stated, staggered arrangement has been found useful to place the rectangular ribs on the horizontal plates. It does not matter that cooling or heating is aspired in the ducts where the working fluid is used, the staggered baffles are placed to improve the efficiency of thermal process as similar approach proposed by Kaewkohkiat et al. (2017). Staggered rib configuration is applicable for the thermal systems needing periodical flow conditions as in the leading study discussed by Patankar et al. (1977). Consecutive studies for the staggered arrangement of the rectangular ribs have been done about convective heat transfer (Webb and Ramadhyani, 1985, Mayle, 1991, Liu and Wang, 2011, Wongcharee et al., 2011, Desrues et al., 2012, Xie et al., 2013, Marocco and Franco, 2017). In comparison studies done by Promvonge and Thianpong (2008), Skullong et al. (2015), Vanaki and Mohammed (2015), Yang et al. (2017); the staggered and symmetrical formations have been given together for the same rib design.

Different channels having both various rib heights and rib spacings have been considered for the comparison at $\operatorname{Re}=10000$. The contour graphics of heat transfer and flow characteristics were occasionally encountered in the past studies. For this reason, the graphics for temperature (T) contours, pressure $(\mathrm{P})$ distributions, streamwise velocity components $(\mathrm{u})$ and streamline patterns $(\Psi)$ have been presented for understanding the problem. The aim of the study is to obtain the ducts having acceptable thermal performance factor values by taking Nusselt numbers and pressure coefficients into account depending on the graphics for thermal and hydraulic characteristics.

\section{COMPUTATIONAL STUDY}

The rectangular ribs have been mounted on the opposite plates of the horizontal duct for the staggered arrangement. Heat transfer augmentation due to the ribs has been examined for two dimensional analyses by employing k- $\omega$ SST model. One of the ribbed channels was indicated in Fig. 1 as an example.

The distance between the plates, $\mathrm{H}$, is taken as 0.05 $\mathrm{m}$. All dimensions in the study have been given in the non-dimensional form, $\mathrm{L}^{\prime}=\mathrm{L} / \mathrm{H}=20$, as dividing by the channel height for its length. The constant rib width is w' $=\mathrm{w} / \mathrm{H}=0.1$ as shown. The range for the rib height values is $0.1 \leq h^{\prime}=h / H \leq 0.3$ while the spacing between two successive ribs is from $\mathrm{S}^{\prime}=\mathrm{S} / \mathrm{H}=0.5$ to 1 . What is more, a rib of any wall has been centered in compliance with the ones on the opposite wall. The ribs were placed after L' $=10$ for the effect of fully developed flow (Cengel and Cimbala, 2006). Ten different models have been used by taking the aforementioned dimensions into consideration.

Thermal and hydraulic characteristics of the parallel plates have been discussed at $\operatorname{Re}=\mathrm{U}_{\infty} \mathrm{D}_{\mathrm{H}} / \mathrm{V}=10000$ for constant thermophysical properties. Computational Fluid Dynamics (CFD) is widely used for the engineering applications including problems of fluid mechanics, heat transfer and thermodynamics.

The flow domains for ten models have been utilized for the numerical analyses. For the following pace, the proper grid system is constituted by considering the reference parameters. The boundary conditions for the flow field have been defined as represented in Fig. 2. Uniform velocity inlet for the fluid at $300 \mathrm{~K}$ and pressure outlet were determined. No-slip boundary condition has been defined for the plates which are kept at $400 \mathrm{~K}$ as can be seen.

Generation of the grid structure depends on various components and parameters. Accordingly, a corre-

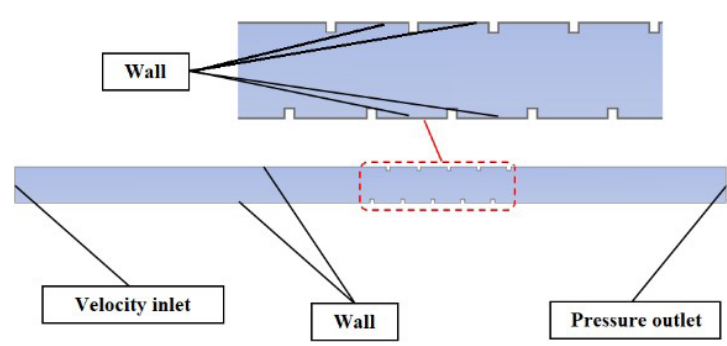

Figure 2. Boundary conditions 
Table 1.Nusselt numbers attained from different turbulence models (Goktepeli et al., 2020)

\begin{tabular}{cc}
\hline Turbulence model & Nu \\
\hline$k-\varepsilon$ Realizable & 97.63 \\
$k-\varepsilon$ Re-Normalisation Group (RNG) & 115.37 \\
$k-\omega$ Shear Stress Transport (SST) & 86.43 \\
$k$ - $\omega$ Standard & 87.55 \\
\hline
\end{tabular}

lation of Eq. 1 has been utilized for the result validation in the smooth duct (Matsubara et al., 2016). Therefore, the numerical solution has to have no dependence of the grid system.

$$
N u=0.021 \operatorname{Pr}^{0.5} \operatorname{Re}^{0.8}
$$

The result of the correlation for Nusselt number $(\mathrm{Nu}=$ $\mathrm{h} \mathrm{D}_{\mathrm{H}} / \mathrm{k}$ ) gives the value of $\mathrm{Nu}=84.19$ for Reynolds number of the present study. The obtained value has been taken as reference for the smooth duct. The numerical results of various turbulence models at $\operatorname{Re}=10000$ have been compared with that of the aforementioned correlation (Goktepeli et al., 2020). The results from the independence test of turbulence models have been indicated in Table 1 . The closest result to that of the reference correlation has been attained via $k-\omega$ Shear Stress Transport (SST) turbulence model.

Table 2.Nusselt numbers obtained for different grid numbers via k- $\omega$ SST turbulence model (Goktepeli et al., 2020)

\begin{tabular}{cc}
\hline Grid number & $N u$ \\
\hline $3 \times 10^{5}$ & 87.01 \\
$4.3 \times 10^{5}$ & 86.46 \\
$6.6 \times 10^{5}$ & 86.43 \\
$8.6 \times 10^{5}$ & 86.52 \\
$1.16 \times 10^{6}$ & 86.53 \\
\hline
\end{tabular}

Nusselt numbers have been obtained by employing k- $\omega$ SST turbulence model as shown in Table 2 for the numerical analyses with different grid numbers (Goktepeli et al., 2020). Although the numerical results were approximate for the considered grid numbers, the nearest result has been provided by using $6.6 \times 10^{5}$ elements based on that of the correlation.

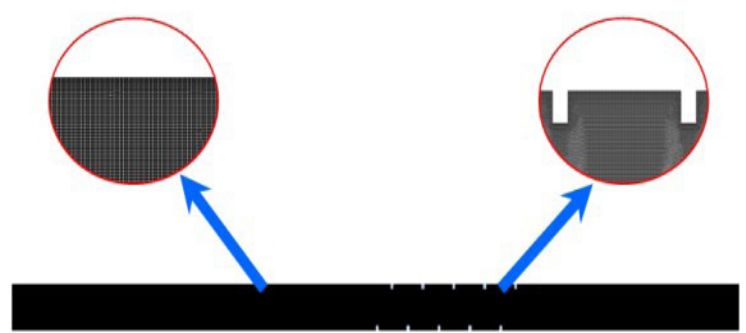

Figure 3. The meshed model
The values for mesh quality are agreeable with the ones of the proposed ranges (Anonymous, 2009). Thinner grid elements have been intensified in the vicinity of the ribbed section to consider boundary layer. Total thickness inflation for the first ten layers and edge sizing has been implemented. A meshed flow domain including the detailed views of Fig. 3 was presented.

Once both appropriate grid structure and turbulence model were ascertained, all of the numerical simulations were conducted based on the results of the independence studies. Continuity and momentum equations have been solved. Reynolds-Averaged Navier-Stokes (RANS) equations are attained with the time-averaged versions of the aforementioned equations. For the incompressible flow, the continuity and the momentum equations were seen in Eqs. (2) and (3), respectively (Anonymous, 2009):

$$
\begin{aligned}
& \frac{\partial \overline{u_{i}}}{\partial x_{i}}=0 \\
& \frac{\partial \bar{u}_{i}}{\partial t}+\frac{\partial\left(\bar{u}_{i} \bar{u}_{j}\right)}{\partial x_{j}}=-\frac{1}{\rho} \frac{\partial \bar{p}}{\partial x_{i}}-\frac{\partial \tau_{i j}}{\partial x_{j}}+\frac{\partial}{\partial x_{j}}\left(v \frac{\partial \bar{u}_{i}}{\partial x_{j}}\right)
\end{aligned}
$$

Energy equation, also in Eq. (4), is solved for heat transfer cases (Anonymous, 2009):

$$
\frac{\partial}{\partial t}(\rho E)+\frac{\partial}{\partial x_{i}}\left[u_{i}(\rho E+p)\right]=\frac{\partial}{\partial x_{j}}\left[\left(k+\frac{C_{p} \mu_{t}}{\operatorname{Pr}_{t}}\right) \frac{\partial T}{\partial x_{j}}+u_{i}\left(\tau_{i j}\right)_{e f f}\right]+S_{h}
$$

The terms are turbulent stresses included in the solution via turbulence modeling where Eqs. (5) and (6) are used (Anonymous, 2009):

$$
\begin{array}{r}
\frac{\partial}{\partial t}(\rho k)+\frac{\partial}{\partial x_{i}}\left(\rho k u_{i}\right)=\frac{\partial}{\partial x_{j}}\left[\Gamma_{k} \frac{\partial k}{\partial x_{j}}\right]+\tilde{G}_{k}-Y_{k}+S_{k} \\
\frac{\partial}{\partial t}(\rho \omega)+\frac{\partial}{\partial x_{i}}\left(\rho \omega u_{i}\right)=\frac{\partial}{\partial x_{j}}\left[\Gamma_{\omega} \frac{\partial \omega}{\partial x_{j}}\right]+G_{\omega}-Y_{\omega}+D_{\omega}+S_{\omega}
\end{array}
$$

Based on the equations, $\mathrm{k}$ and $\omega$ are turbulent kinetic energy and specific dissipation rate, respectively. What is more, $\tilde{G}_{k}$ is the generation of the turbulent kinetic energy resulting from the mean velocity gradients and the specific dissipation rate generation is given as $G_{\omega}$. The effective diffusivity values for $k$ and $\omega$ are $\Gamma_{k}$ and $\Gamma_{\omega}$, respectively. Furthermore, $Y_{k}$ and $Y_{\omega}$ are dissipation of $k$ and $\omega$ as a 
result of turbulence. The cross-diffusion term is $\mathrm{D}_{\omega}$ while $S_{k}$ and $S_{\omega}$ are the user-defined source terms (Goktepeli et al., 2020).

For the simulations, the maximum number of iterations per time step was twenty for the time step of $0.0068 \mathrm{~s}$. However, the iterations terminate for the analysis when the residuals of the equations are balanced to $10^{-8}$. The dimensionless wall distance was $y^{+}=u^{*} y / v \cong 1$ (Goktepeli et al., 2020).

\section{RESULTS AND DISCUSSION}

Heat transfer and fluid flow characteristics have been presented for $\operatorname{Re}=10000$ in Figs. $4-13$ and Table 3 after conducting the numerical analyses. These aforementioned results have been discussed for relevant subheadings of temperature $(\mathrm{T})$ contours, pressure $(\mathrm{P})$ distributions, streamwise velocity components $(\mathrm{u})$, streamline patterns $(\Psi)$, normalized mean Nusselt numbers $(\overline{N u})$ together with local Nusselt numbers $(\mathrm{Nu})$, local pressure coefficients $\left(C_{p}\right)$ and thermal performance factor $(\eta)$ values. The top-down arrangement for the figures of temperature $(\mathrm{T})$ contours, pressure $(\mathrm{P})$ distributions, streamwise velocity components $(\mathrm{u})$, streamline patterns $(\Psi)$ is smooth channel, the ribbed channel with $\mathrm{S}^{\prime}=0.5$ and $\mathrm{h}^{\prime}=$ 0.1 , the ribbed channel with $S^{\prime}=0.5$ and $h^{\prime}=0.2$, the ribbed channel with $\mathrm{S}^{\prime}=0.5$ and $\mathrm{h}^{\prime}=0.3$, the ribbed channel with $S^{\prime}=0.75$ and $h^{\prime}=0.1$, the ribbed channel with $S^{\prime}=$ 0.75 and $h^{\prime}=0.2$, the ribbed channel with $S^{\prime}=0.75$ and $h$ $=0.3$, the ribbed channel with $\mathrm{S}^{\prime}=1$ and $\mathrm{h}^{\prime}=0.1$, the ribbed channel with $\mathrm{S}^{\prime}=1$ and $\mathrm{h}^{\prime}=0.2$ and the ribbed channel with $\mathrm{S}^{\prime}=1$ and $\mathrm{h}^{\prime}=0.3$.

\section{Temperature Contours}

Time-averaged results of temperature $\langle\mathrm{T}\rangle$ distributions have been given based on the numerical analyses in Fig. 4 . Fluid at $300 \mathrm{~K}$ has been supposed to enter the horizontal parallel plates of duct maintained at $400 \mathrm{~K}$. The maximum and the minimum values of temperature contours were as $400 \mathrm{~K}$ and $300 \mathrm{~K}$, respectively for the investigated problem. Due to the fact that the temperature distributions have been differently observed in terms of various types of the ducts, changes of the heat transfer characteristics are obviously sighted. Nonetheless, the ribbed ducts are more dominant over the smooth plates from the point of heat transfer. In the general sense, this situation is explained by temperature alterations seen in the vicinity of to the wall. Due to this reason, the channels with the ribs are very effective in terms of heat transfer when compared to the smooth duct. As a result of the interaction between fluid and solid regions, flow separation has been seen because of the first rib at the upstream. Another explanation on this case is the breaking down of both hydrodynamic and thermal boundary layers with

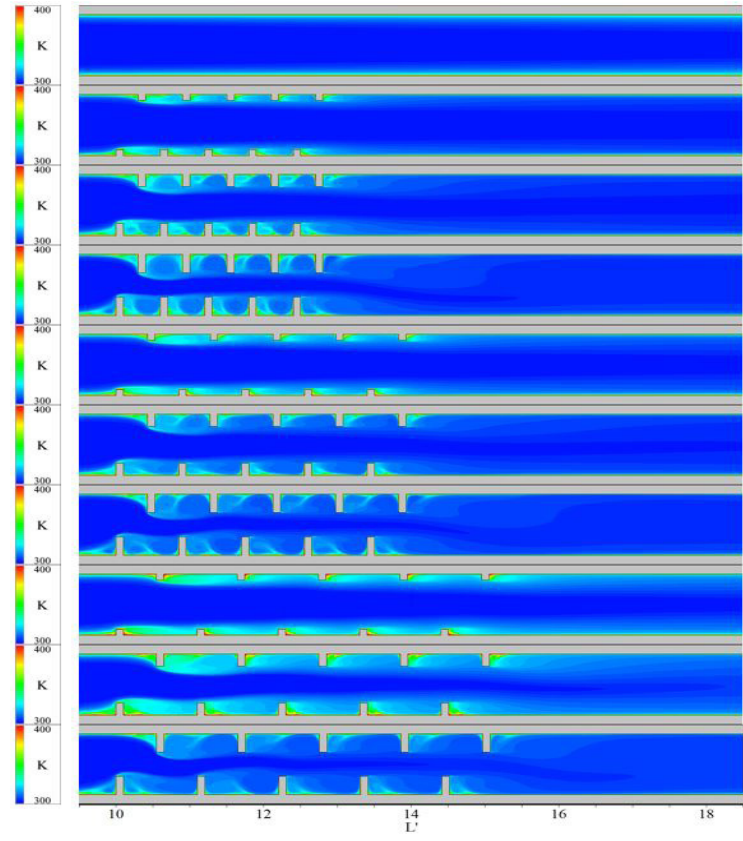

Figure 4. Temperature $(\mathrm{T})$ contours at $\mathrm{Re}=10000$

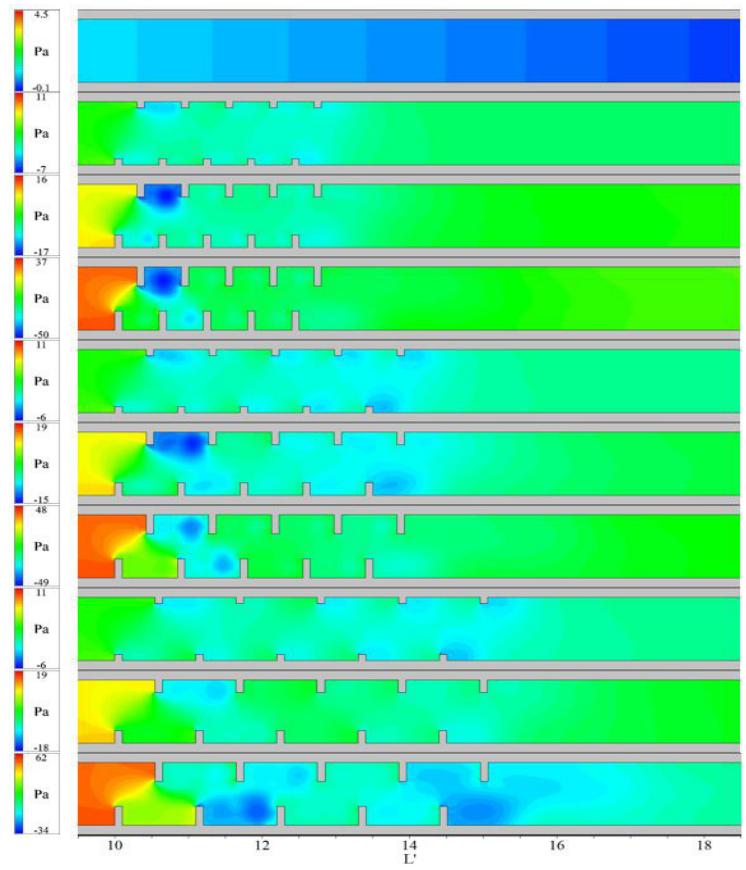

Figure 5. Pressure $(\mathrm{P})$ distributions at $\mathrm{Re}=10000$

the effect of the ribs. Instantaneous changes in temperature values have been seen due to complex flow structure in these zones. Heat transfer has been substantially augmented as explained. It has been beheld for all cases including the ribbed channels. Increase of fluid temperature along the centerline has been occurred by virtue of the reduction of cross-section triggering the flow mixing. What is more, surface area for heat transfer has been become wider by the ribs. It is known that this circumstance positively affects heat transfer enhancement. Even 


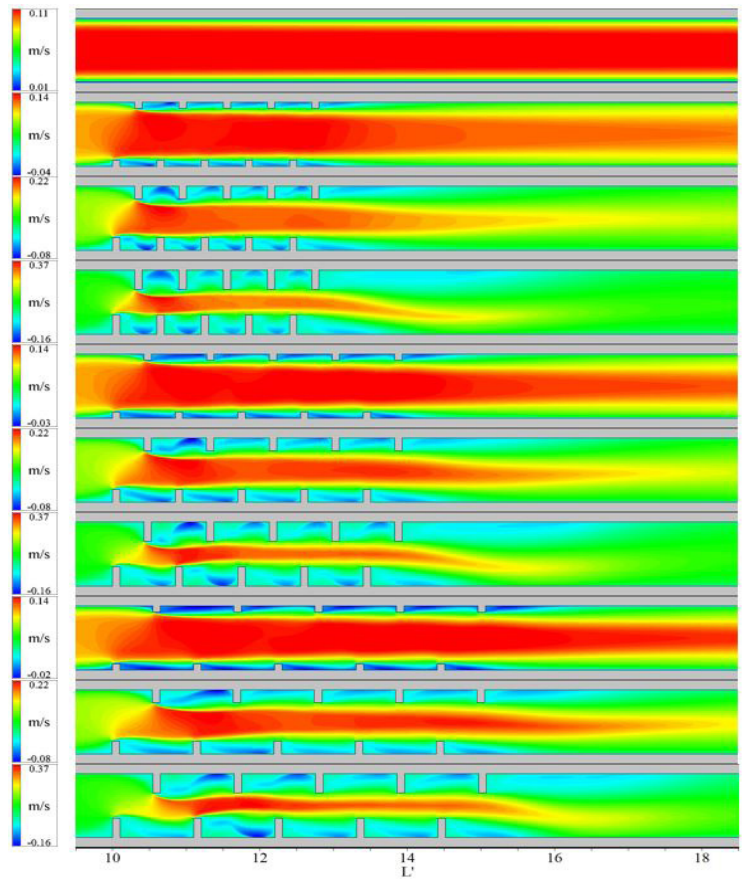

Figure 6. Streamwise velocity components $(\mathrm{u})$ at $\mathrm{Re}=10000$

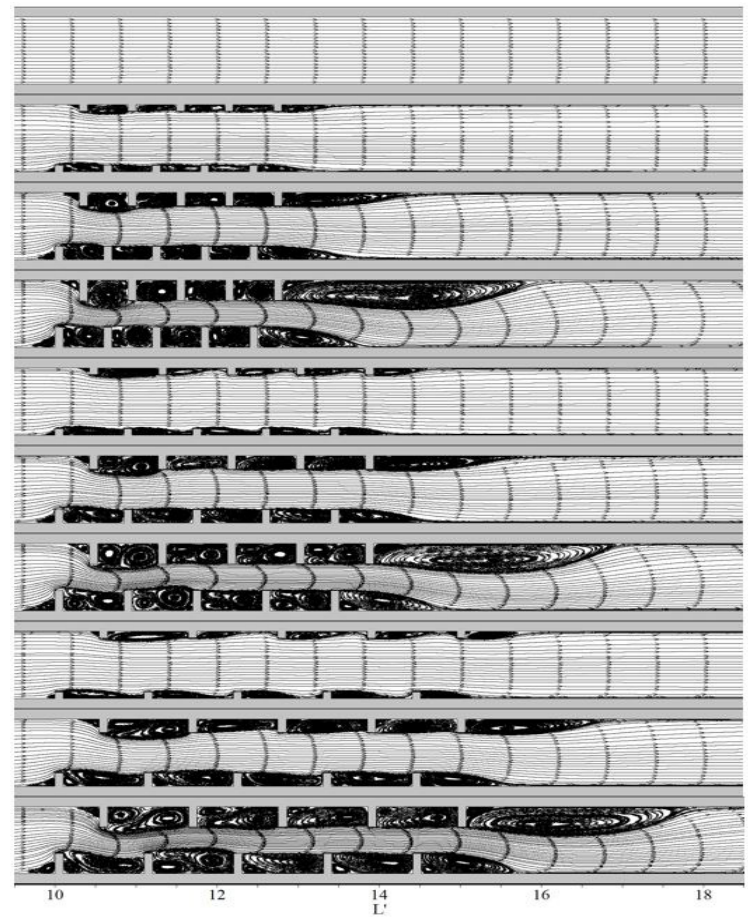

Figure 7. Streamline patterns $(\Psi)$ at $\operatorname{Re}=10000$

though placing the longer rib to enhance the effect of flow separation, the rib height is limitative in terms of the margin of both plates. Another constraint to be born in mind is pressure drop for the design of the heat transfer system. In the present case, changing the distance between two successive ribs has been considered to augment the heat transfer. Based on the present results, heat transfer has been increased with the increment of the margin between the ribs owing to the fact that fluid flow simply steps in the gap to generate recirculation. It has been observed with temperature distributions presented in Fig. 4. Moreover, the contact zones between fluid and solid regions have been enlarged by ascending the margin thereafter the flow reattachment. For instance, it is difficult for fluid flow to move in the region covered by two ribs due to narrower spacing. It can be observed in Fig. 4 that only one circulation zone occurs when the ribs are closer enough to each other. While the distance is increased, two circulation zones can be observed and heat transfer coefficient is increased with this manner. As seen in Fig. 7, recirculating regions have also been observed via streamline patterns.

\section{Pressure Distributions}

Time-averaged results for pressure distributions have been indicated in Fig. 5. Pressure drop for the smooth duct was less when compared to the ribbed model as anticipated. There is gradual drop for pressure values through the smooth channel. Furthermore, pressure values have been increased by mounting the ribs on the plates. However, the effect of the rib spacing on pressure values is less than that of other parameters. For this reason, there is strong decrease in pressure values for all cases with the ribbed ducts. The section that rapid decrement of pressure values took place is broadly the wake region of the first rib on the upper plate. Especially, this is the minimum pressure region for $\mathrm{S}^{\prime}=0.5$. When the rib spacing has been increased step by step, the secondary minimum pressure regions have been seen in the wakes of different ribs based on the position. After the second ribs, the increment of pressure values has been observed and the values approached to the average ones of the legend bar. Accordingly, transitional recovery for pressure values has been obtained due to the flow rate decrement resulting from the second ribs. The recovery in pressure values was earlier for $\mathrm{S}^{\prime}=0.5$ and its trend was effectual up to the end of the ribbed section. However, pressure values decreased around the last rib of the lower plate. After passing the ribbed zone, average pressure values were dominant.

\section{Streamwise Velocity Components}

Time-averaged results of streamwise velocity components $<\mathrm{u}>$ have been presented in Fig. 6. The maximum values for the streamwise velocity components have been attained at the outside of boundary layer for the smooth duct. There is decrement in the velocity values for the regions affected by the boundary layer owing to no-slip condition on the plate. Rotational flow has not been obtained in the absence of the ribs. As can be seen from the graphics, the channel cross-section is downsized by the virtue of the ribs mounted on the 

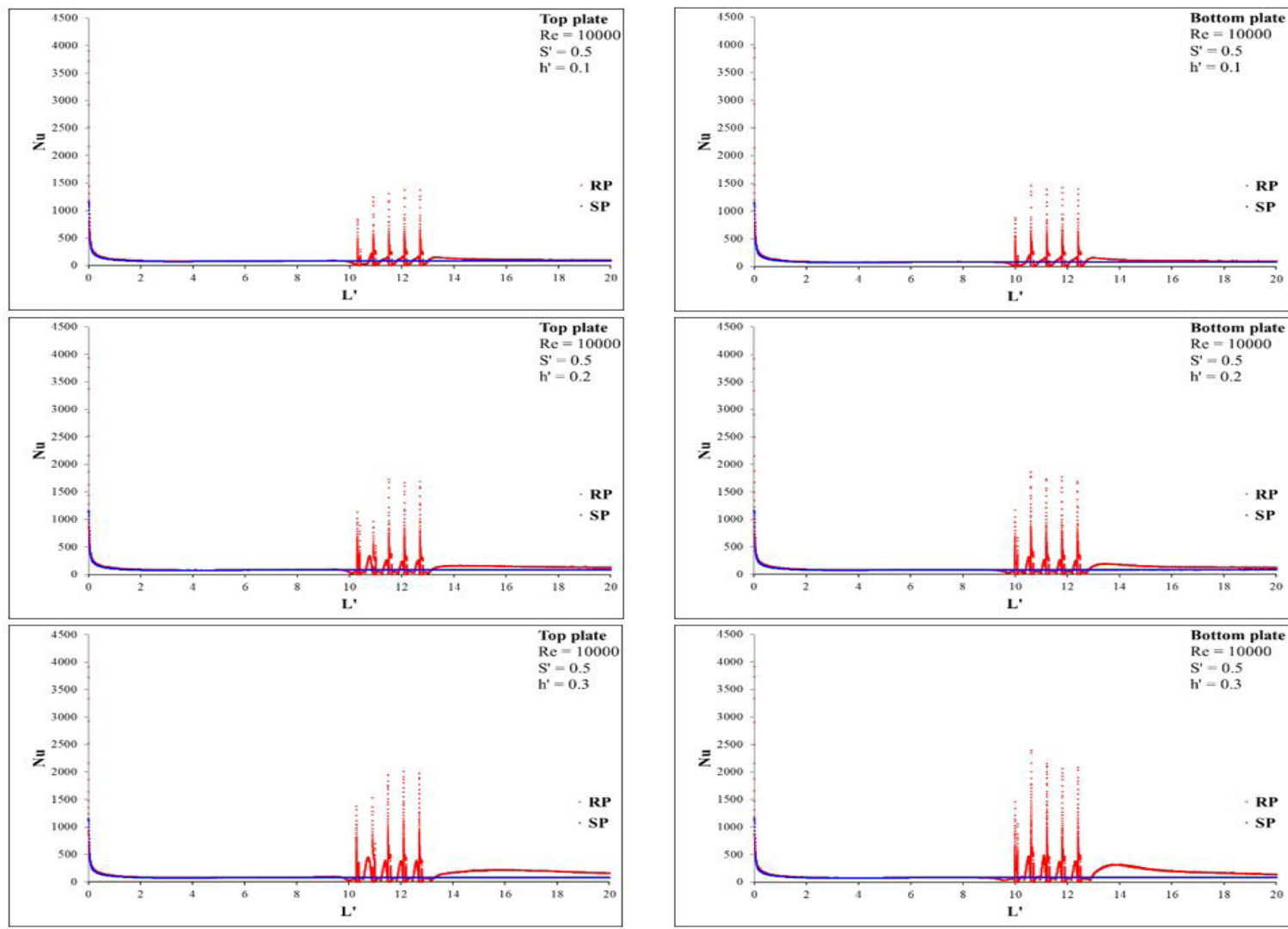

Figure 8. Local Nusselt numbers $(\mathrm{Nu})$ through the channels for varying rib heights of $\mathrm{S}^{\prime}=0.5$ at $\mathrm{Re}=10000$
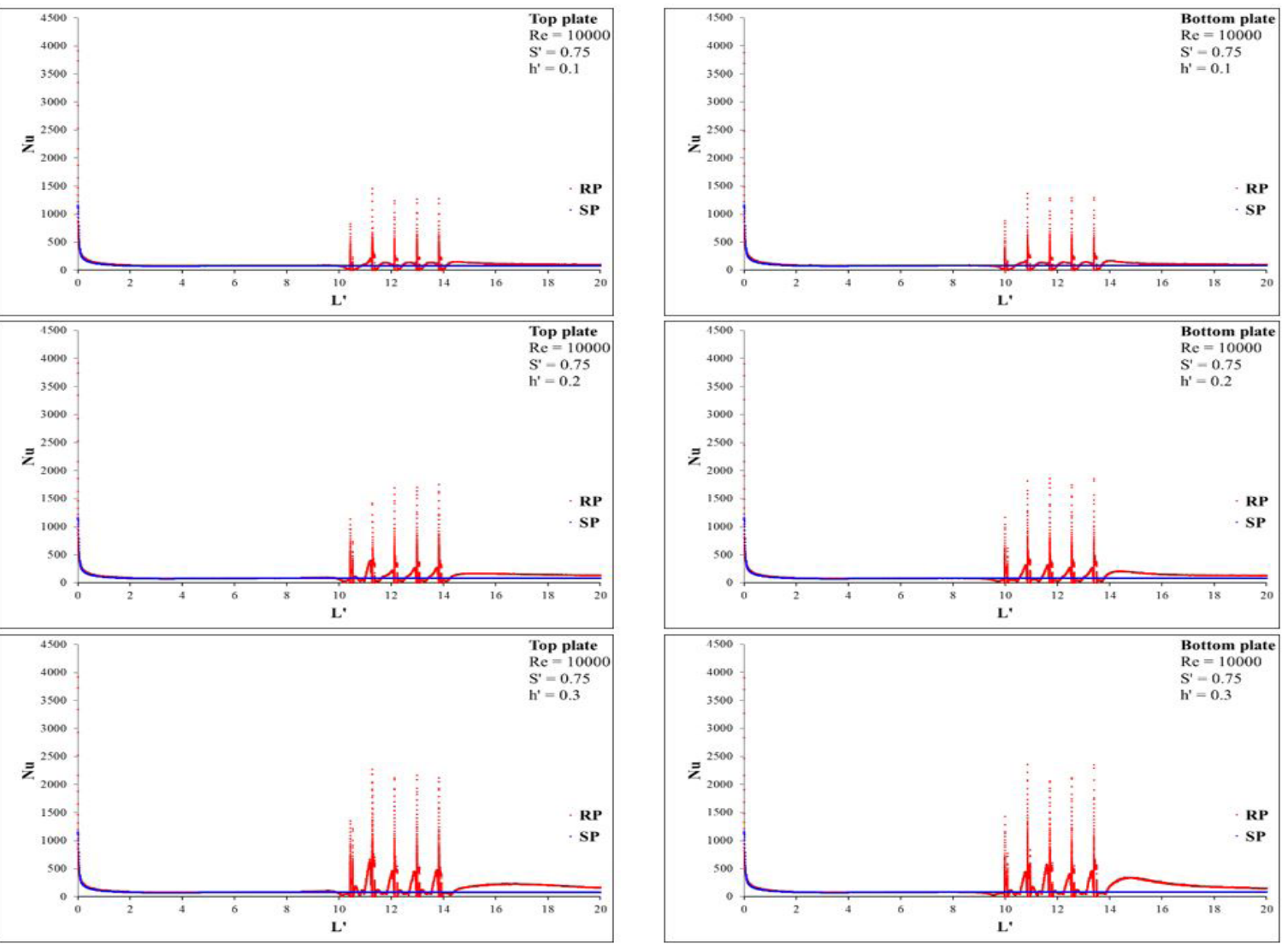

Figure 9. Local Nusselt numbers $(\mathrm{Nu})$ through the channels for varying rib heights of $\mathrm{S}^{\prime}=0.75$ at $\mathrm{Re}=10000$ 

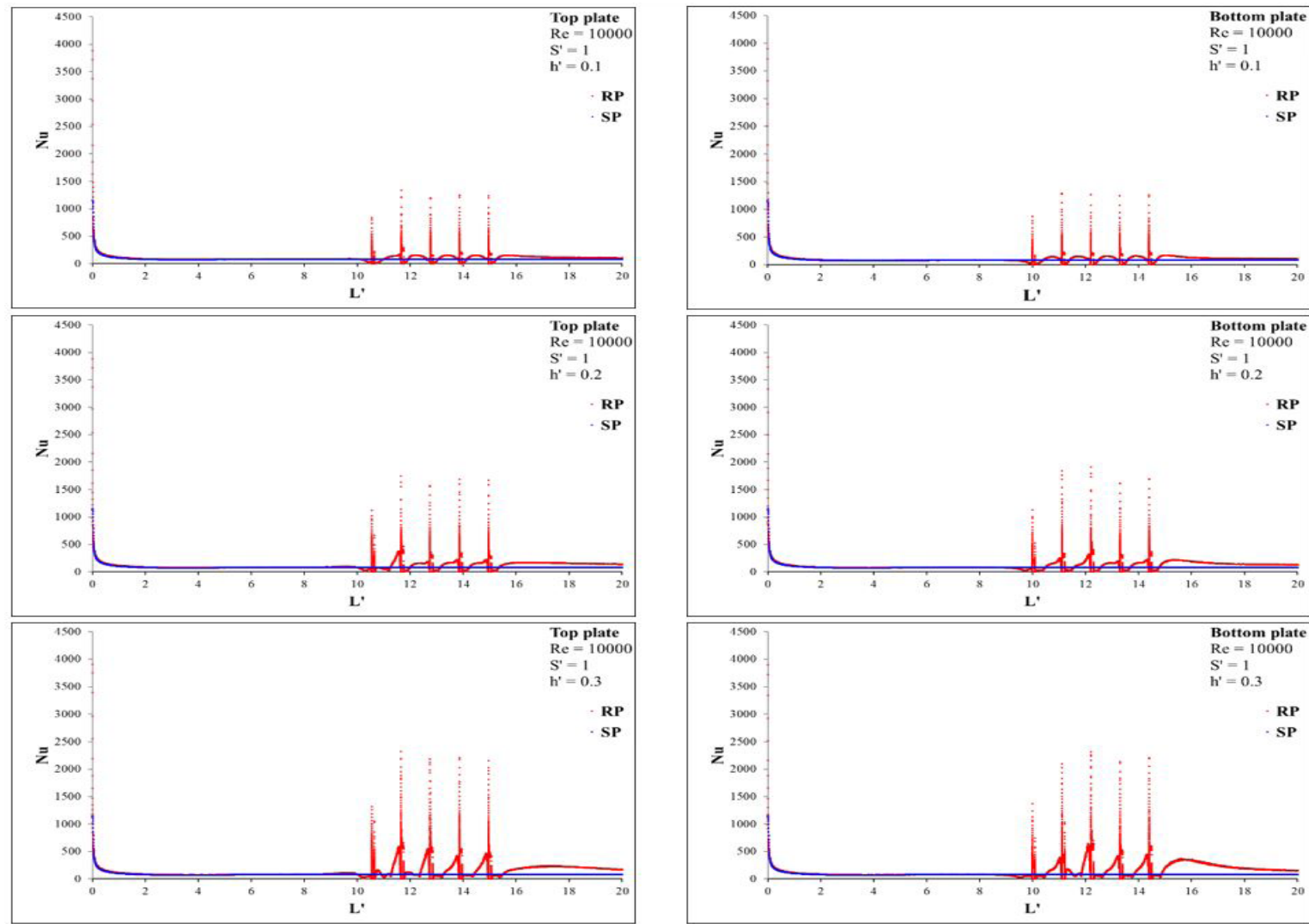

Figure 10.Local Nusselt numbers $(\mathrm{Nu})$ through the channels for varying rib heights of $\mathrm{S}^{\prime}=1$ at $\operatorname{Re}=10000$
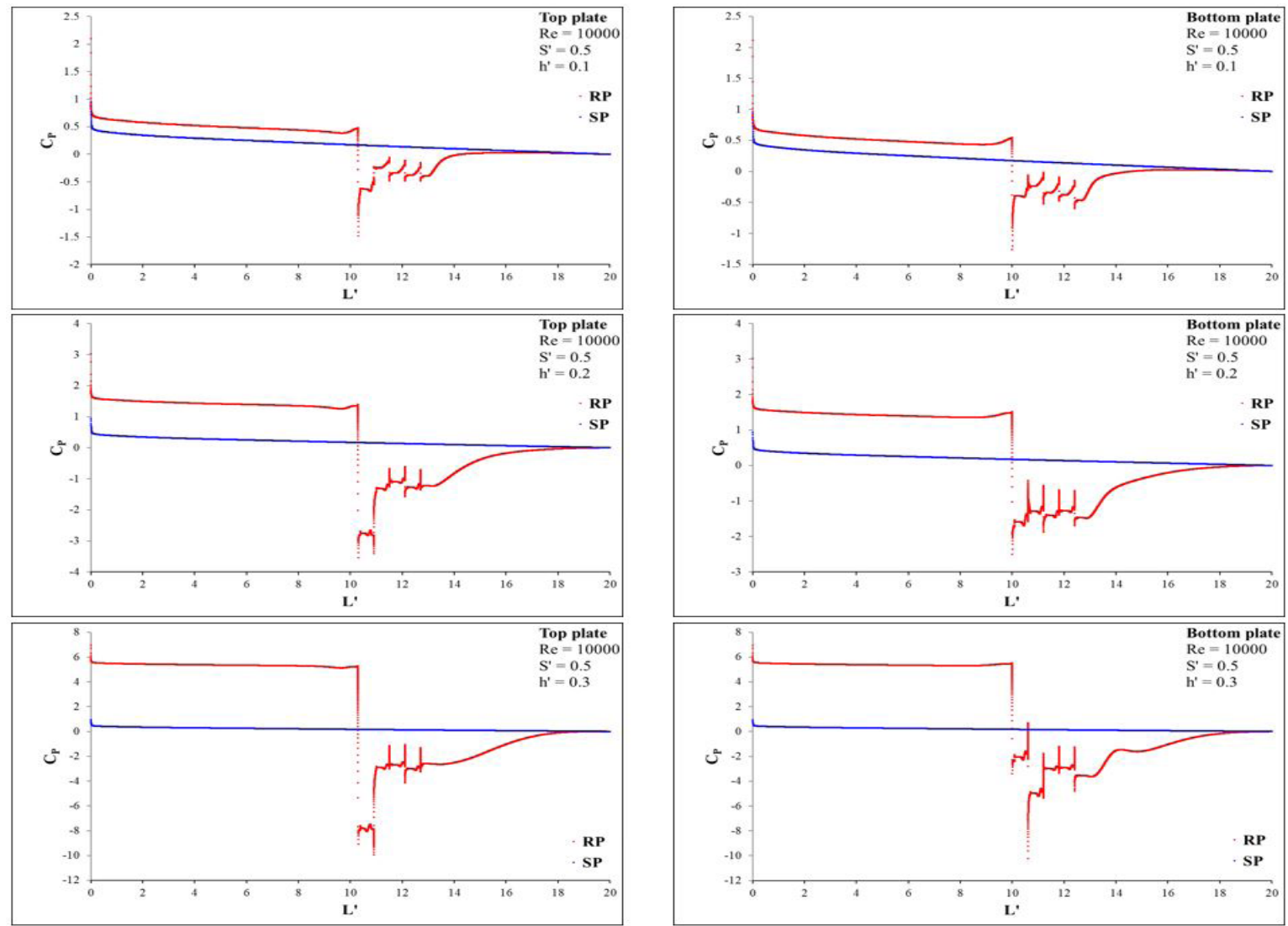

Figure 11. Local pressure coefficients $\left(C_{P}\right)$ through the channels for varying rib heights of $S^{\prime}=0.5$ at $\operatorname{Re}=10000$ 

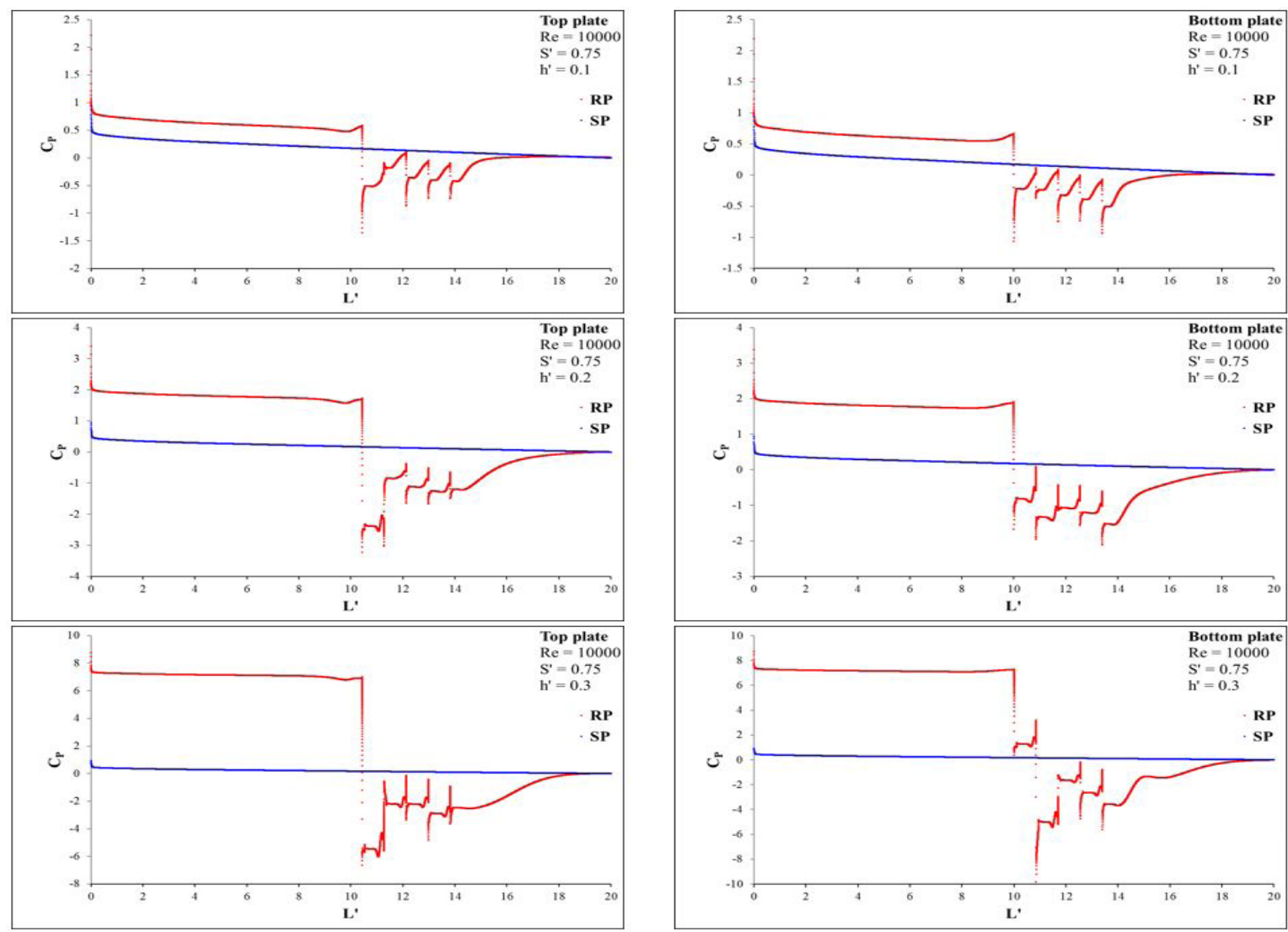

Figure 12. Local pressure coefficients $\left(C_{P}\right)$ through the channels for varying rib heights of $S^{\prime}=0.75$ at $\operatorname{Re}=10000$
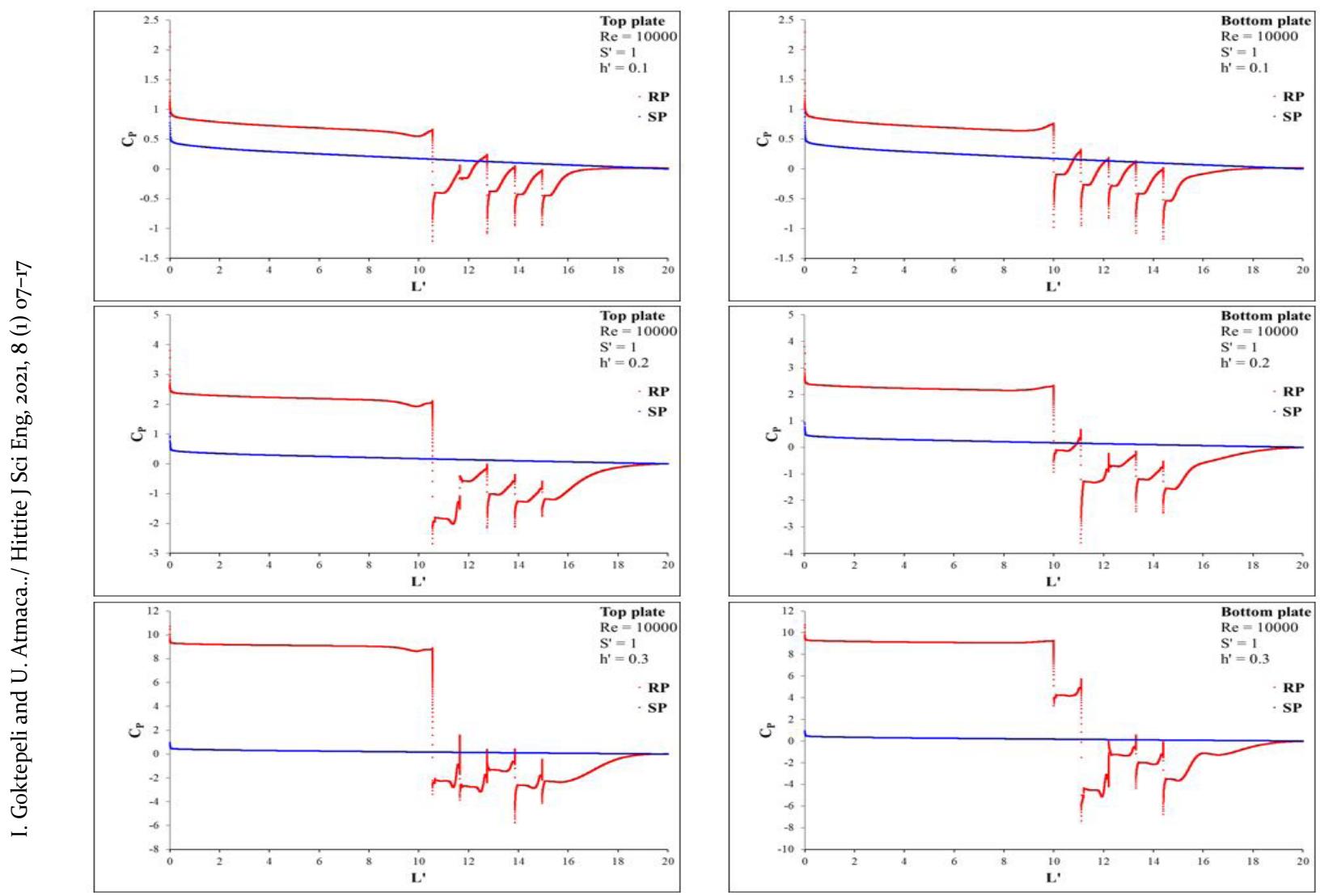

Figure 13. Local pressure coefficients $\left(C_{P}\right)$ through the channels for varying rib heights of $S^{\prime}=1$ at $\operatorname{Re}=10000$ 
walls. Due to this reason, negative velocity components have been attained in the ribbed region. Thus, there is increase for the velocity values observed because of the decrement of the cross-sectional area in the duct. It is the result of the flow separation triggered by the first rib on both upper and lower walls. Asymmetrical flow structure has been obtained as a result of staggered arrangement. In case of the forward-facing step flow where after the flow separates from the walls, it reattaches to the upstream corner of the rib and it is again separated from that point (Goktepeli et al., 2021). In the regions where flow separated, the rotational flows have been seen at the upstream of the rib. This is the forward-facing step flow at the upstream of the rib, the cavity flow between the ribs and the backward-facing step flow at the downstream of the rib (Yemenici and Umur, 2016). When it comes to cavity flow, rotational flow that is more energetic between the first two ribs comparatively has lost its effect for the gap between the other ones. In terms of the most cases, the regions for the maximum velocity values obtained were formed by the first two ribs and the upper side of the centerline. Nevertheless, there is not much alteration for the velocity values while the spacing between the ribs is enlarged. The region of maximum streamwise velocity values observed was between the other ribs with respect to the increase of rib spacing.

\section{Streamline Patterns}

Time-averaged results of streamline patterns $\langle\Psi\rangle$ have been exhibited in Fig. 7. Uniform flow structure has been obtained without the additional elements diminishing the flow for the smooth channel. Chaotic flow structure has been considerably obtained in the existence of the ribs mounted on the walls of the duct. However, the width between the ribs has effect on complexness degree of flow field.

Rotational flow has been seen between first ribs in a row due to flow separation considerably affected by them. Since the leading element to distort the flow was the first rib, larger vortices have been attained in the wake of the first one for both plates. The separated flow produces a lower pressure region at the downstream of the first ribs as in Fig. 7. Periodical flow has been seen because of the rib arrangement and the intensity of eddies was less than the first ones. What is more, the required distance for the flow recovery does not vary with the rib spacing.

\section{Local And Mean Nusselt Number Values}

Nusselt number values through both ribbed and smooth channels have been given for the comparison in Figs 8-10. On the graphics, the abbreviations of "RP" and "SP" are for the ribbed and smooth channels, respectively. Nusselt number values have been presented for the overall duct length. Moreover, in Table 3, Nusselt numbers for the ribbed channels have been compared by using the values of the smooth plate.

As given in Figs. 8-10, Nusselt number values of both smooth and ribbed plates coincided for $0 \leq L^{\prime} \leq 9$. The values of Nusselt numbers tend to increase suddenly after the first rib. The value around the first rib is relatively lower than those of the rest of the ribs. Nonetheless, the values have decreased between the successive ribs. The peak values due to each rib have increasing effect on the mean Nusselt number. Therefore, heat transfer has been augmented. After passing the fifth rib, the values of Nusselt numbers have sharply dropped and re-increased. Flow recovery has been observed in the vicinity of the outlet. This recovery delays owing to increasing all parameters. As clearly seen for all of the cases, the asymmetrical distributions have been observed because of the staggered arrangement. The minimum values have been seen at the reattachment points between the ribs.

Moreover, the mean Nusselt numbers of the ribbed ducts have also been compared with that of smooth channel in terms of normalized values. Heat transfer has been enhanced by mounting the ribs as can be seen in Table 3 where mean Nusselt numbers for the ribbed ducts against the value of the smooth plate have been given. Heat transfer increment varies based on the effect of the geometrical dimensions related with the ribs such as height, spacing etc. Nusselt numbers have been attained at higher values for increasing rib height and the highest increment has been observed when it is from $h^{\prime}=0.2$ to $h^{\prime}=0.3$, respectively. The relative change for the Nusselt number values are \% 25 for $\mathrm{S}^{\prime}$ $=0.5, \% 28.3$ for $\mathrm{S}^{\prime}=0.75$ and $\% 29.4$ for $\mathrm{S}^{\prime}=1$ when the rib heights are increased from $h^{\prime}=0.2$ to $h^{\prime}=0.3$. In this study, another parameter enhancing the convective heat transfer is the rib spacing in terms of Nusselt number values. The spacing effect on Nusselt number values is clearly seen from $\mathrm{S}^{\prime}=0.5$ to $\mathrm{S}^{\prime}=0.75$ as can be seen in the chart. The relative changes for the Nusselt numbers are \% 2.1 for h' $=0.1$, \% 2.3 for $h^{\prime}=0.2$ and $\% 4.6$ for $h^{\prime}=0.3$ when the rib spacing values

Table 3.Normalization of mean Nusselt numbers $(\overline{N u})$ at $\operatorname{Re}=10000$

\begin{tabular}{cc}
\hline Ribbed channels & $R e=10000$ \\
\hline$S^{\prime}=0.5$ and $h^{\prime}=0.1$ & 1.198 \\
$S^{\prime}=0.5$ and $h^{\prime}=0.2$ & 1.414 \\
$S^{\prime}=0.5$ and $h^{\prime}=0.3$ & 1.775 \\
$S^{\prime}=0.75$ and $h^{\prime}=0.1$ & 1.223 \\
$S^{\prime}=0.75$ and $h^{\prime}=0.2$ & 1.446 \\
$S^{\prime}=0.75$ and $h^{\prime}=0.3$ & 1.856 \\
$S^{\prime}=1$ and $h^{\prime}=0.1$ & 1.229 \\
$S^{\prime}=1$ and $h^{\prime}=0.2$ & 1.443 \\
$S^{\prime}=1$ and $h^{\prime}=0.3$ & 1.867 \\
\hline
\end{tabular}


are increased from $S^{\prime}=0.5$ to $S^{\prime}=0.75$, respectively. However, these changes are relatively less than those of the rib height. In other words, the effect of the rib height is much more than that of the rib spacing in terms of Nusselt number values. The results show that the highest increase of Nusselt number has been obtained for the maximum rib height of $h$ $=0.3$ and the maximum rib spacing of $S^{\prime}=1$ based on that of the smooth plate.

\section{Local Pressure Coefficients}

Local distributions of pressure coefficients have been given as presented in Figs. 11-13. Even though there is no rib mounted for $0 \leq \mathrm{L}^{\prime} \leq 9$, noteworthy difference between the pressure coefficients of both ribbed and smooth plates has been observed. Maximum pressure coefficient was at the duct inlet and the values started to decrease up to the duct outlet. The drop is nearly similar for both plates with $0 \leq L^{\prime} \leq 9$. After the first rib, the local values have suddenly decreased. The initial point of the recuperation region for the pressure coefficients can be postponed with the increment of the rib height and the rib spacing. Enormous influence that increases the pressure coefficients is arisen from the increase of the rib height. It can be induced that the effect of the rib spacing on the pressure coefficients is relatively less.

\section{Values For Thermal Performance Factor}

Although the ratio of Nusselt numbers indicates that how much heat transfer enhancement is obtained, it is not enough for the system design since it does not include any pressure loss term that has to be considered for the envisagement. For this reason, the values for thermal performance factor $(\eta)$ of the ribbed plates have been calculated. Here, $\eta=\left(\mathrm{Nu} / \mathrm{Nu}_{0}\left(\mathrm{f} / \mathrm{f}_{0}\right)^{-1 / 3}\right.$ states the optimum value with respect to the heat transfer augmentation over pressure loss. If the thermal performance factor is $\eta$ $\geq 1$, it means that the ribbed duct can be proposed for increment of heat transfer as pressure drop for the system is tolerated. In the present study, $\mathrm{h}^{\prime}=0.1$ with $\mathrm{S}^{\prime}=$ 0.5 at $\operatorname{Re}=10000$ having $\eta=1.049$ and $h^{\prime}=0.1$ with $S^{\prime}=$ 0.75 at $\operatorname{Re}=10000$ having $\eta=1.019$ are the potential ribbed channels for the recommandation in terms of heat transfer enhancement.

\section{CONCLUSION}

Heat transfer and fluid flow characteristics for the plates have been considered via the staggered ribs at $\mathrm{Re}=10000$, in the present study. By keeping the rib width of $\mathrm{w}^{\prime}=0.1$ constant, the rib height and the rib spacing have been respectively changed as $0.1 \leq \mathrm{h}^{\prime} \leq 0.3$ and $0.5 \leq \mathrm{S}^{\prime} \leq 1$. The results have been summarized as:

More chaotic flow structure has been observed by using the ribs for the staggered arrangement. Therefore, heat transfer has been augmented as a result of increasing heat transfer coefficient due to the flow mixing.
Increasing the height of the rib and the spacing between the ribs separately, has an increasing effect on Nusselt number. Here, the rib height is more dominant than the rib spacing in terms of heat transfer enhancement.

- $\quad$ Pressure drop has been observed owing to the increment of the rib height and the spacing between the ribs. However, as in Nusselt number, the rib height is more effective than the rib spacing when it comes to pressure loss.

- $\quad$ Since increasing the rib height and the rib spacing increases both heat transfer by convection and pressure drop, performance criterion has to be calculated for the optimization. To sum up, h' $=0.1$ with $S^{\prime}=0.5$ having $\eta=1.049$ and $h^{\prime}=0.1$ with $S^{\prime}=0.75$ having $\eta=1.019$ have been advised since thermal performance factor values are $\eta \geq 1$, in the present study at $\operatorname{Re}=10000$.

\section{ACKNOWLEDGMENTS}

This study has been supported by Academic Staff Training Program (OYP) with the project number of 2018OYP-046.

\section{NOMENCLATURE}

$\begin{array}{lll}\text { CFD } & : & \text { Computational Fluid Dynamics } \\ \mathrm{C}_{\mathrm{p}} & : & \text { Pressure coefficient } \\ \mathrm{D}_{\mathrm{H}} & : & \text { Hydraulic diameter }[\mathrm{m}] \\ \mathrm{D}_{\omega} & : & \text { Cross-diffusion term } \\ \mathrm{E} & : & \text { Energy [J] } \\ \mathrm{f} & : & \text { Friction factor } \\ \mathrm{G}_{\mathrm{k}} & : & \text { Generation of turbulence kinetic energy } \\ \mathrm{G}_{\omega} & : & \text { Generation of dissipation rate } \\ \mathrm{h} & : & \text { Height, rib height [m] } \\ \mathrm{H} & : & \text { Distance between the plates }[\mathrm{m}] \\ \mathrm{k} & : & \text { Thermal conductivity coefficient }[\mathrm{W} / \mathrm{mK}] \\ & & \text { turbulence kinetic energy [m2/s2] } \\ \mathrm{L} & : & \text { Length [m] } \\ \mathrm{Nu} & : & \text { Nusselt number } \\ \mathrm{P} & : & \text { Pressure [Pa] } \\ \mathrm{RANS} & : & \text { Reynolds-Averaged Navier-Stokes } \\ \mathrm{Re} & : & \text { Reynolds number } \\ \mathrm{RNG} & : & \text { Re-Normalization Group } \\ \mathrm{RP} & : & \text { Ribbed plate } \\ \mathrm{S} & : & \text { Ribspacing [m], user-defined source term } \\ \mathrm{SP} & : & \text { Smooth plate } \\ \mathrm{SST} & : & \text { Shear Stress Transport } \\ \mathrm{t} & : & \text { Time [s] } \\ \mathrm{T} & : & \text { Temperature [K] } \\ \mathrm{u} & : & \text { Streamwise velocity component }[\mathrm{m} / \mathrm{s}] \\ \mathrm{U} & : & \text { Free-stream velocity [m/s] } \\ \mathrm{w} & : & \text { Width, rib width [m] } \\ \mathrm{y}^{+} & : & \text {Dimensionless wall distance } \\ \mathrm{Y} & : & \text { Dissipation due to turbulence } \\ \mathrm{T} & : & \text { Effective diffusivity } \\ \eta & : & \text { Thermal performance factor } \\ & & \end{array}$




$\begin{array}{ll}\mu & : \\ \nu & : \\ \rho & : \\ \tau & : \\ \Psi & : \\ \omega & :\end{array}$

\section{Supercript}

Dimensionless

\section{Subscript}

$0 \quad$ : $\quad$ Smooth plate

\section{REFERENCES}

1. Sundén B. Convective heat transfer and fluid flow physics in some ribbed ducts using liquid crystal thermography and PIV measuring techniques. Heat and Mass Transfer 47 (2011) 899-910

2. Kashyap U, Das K, Debnath BK. Effect of surface modification of a rectangular vortex generator on heat transfer rate from a surface to fluid. International Journal of Thermal Sciences 127 (2018) 61-78.

3. Yang YT, Chen PJ. Numerical optimization of turbulent flow and heat transfer characteristics in a ribbed channel. Heat Transfer Engineering 36 (2015) 290-302.

4. Alfarawi S, Abdel-Moneim S, Bodalal A. Experimental investigations of heat transfer enhancement from rectangula duct roughened by hybrid ribs. International Journal of Thermal Sciences 118 (2017) 123-138.

5. Tiggelbeck S, Mitra NK, Fiebig M. Experimental investigations of heat transfer enhancement and flow losses in a channel with double rows of longitudinal vortex generators. International Journal of Heat and Mass Transfer 36 (1993) 2327-2337.

6. Abdollahi A, Shams M. Optimization of shape and angle of attack of winglet vortex generator in a rectangular channel for heat transfer enhancement. Applied Thermal Engineering 81 (2015) 376-387.

7. Kilicaslan I, Sarac HI. Enhancement of heat transfer in compact heat exchanger by different type of rib with holographic interferometry Experimental Thermal and Fluid Science 17 (1998) 339-346.

8. Sripattanapipat S, Promvonge P. Numerical analysis of laminar heat transfer in a channel with diamond-shaped baffles. International Communications in Heat and Mass Transfer 36 (2009) 32-38.

9. Manca O, Nardini S, Ricci D. Numerical analysis of water forced convection in channels with differently shaped transverse ribs. Journal of Applied Mathematics 2011 (2011) 323485

10. Wongcharee K, Changcharoen W, Eiamsa-Ard S. Numerical investigation of flow friction and heat transfer in a channel with various shaped ribs mounted on two opposite ribbed walls. International Journal of Chemical Reactor Engineering 9 (2011).

11. Ahmed M, Yusoff M, Shuaib N. Effects of geometrical parameters on the flow and heat transfer characteristics in trapezoidal-corrugated channel using nanofluid. International Communications in Heat and Mass Transfer 42 (2013) 69-74.

12. Aslan E, Taymaz I, Islamoglu Y. Finite volume simulation for convective heat transfer in wavy channels. Heat and Mass Transfer 52 (2016) 483-497.

13. Moon MA, Park MJ, Kim KY. Evaluation of heat transfer performances of various rib shapes. International Journal of Heat and Mass Transfer 71 (2014) 275-284
14. Kaewkohkiat Y, Promvonge P, Eiamsa-Ard S. Turbulent periodic flow and heat transfer in a rectangular channel with detached V-baffles. Journal of Engineering Thermophysics 26 (2017) 542-552.

15. Patankar S, Liu C, Sparrow E. Fully developed flow and heat transfer in ducts having streamwise-periodic variations of crosssectional area. Journal of Heat Transfer 99 (1977) 180-186.

16. Webb B, Ramadhyani S. Conjugate heat transfer in a channel with staggered ribs. International Journal of Heat and Mass Transfer 28 (1985) 1679-1687.

17. Mayle RE. Pressure loss and heat transfer in channels roughened on two opposed walls. Journal of Turbomachinery 113 (1991) 60-66.

18. Liu $\mathrm{H}$, Wang J. Numerical investigation on synthetical performances of fluid flow and heat transfer of semiattached ribchannels. International Journal of Heat and Mass Transfer 54 (2011) 575-583.

19. Desrues T, Marty P, Fourmigué J. Numerical prediction of heat transfer and pressure drop in three-dimensional channels with alternated opposed ribs. Applied Thermal Engineering 45 (2012) 52-63.

20. Xie G, Li S, Zhang W, Sunden B. Computational Fluid Dynamics modeling flow field and side-wall heat transfer in rectangular ribroughened passages. Journal of Energy Resources Technology 135 (2013) 042001.

21. Marocco L, Franco A. Direct Numerical Simulation and RANS comparison of turbulent convective heat transfer in a staggered ribbed channel with high blockage. Journal of Heat Transfer 139 (2017) 021701.

22. Promvonge $\mathrm{P}$, Thianpong $\mathrm{C}$. Thermal performance assessment of turbulent channel flows over different shaped ribs. International Communications in Heat and Mass Transfer 35 (2008) 1327-1334.

23. Skullong S, Thianpong C, Promvonge P. Effects of rib size and arrangement on forced convective heat transfer in a solar air heater channel. Heat and Mass Transfer 51 (2015) 1475-1485.

24. Vanaki SM, Mohammed H. Numerical study of nanofluid forced convection flow in channels using different shaped transverse ribs. International Communications in Heat and Mass Transfer 67 (2015) 176-188.

25. Yang W, Xue S, He Y, Li W. Experimental study on the heat transfer characteristics of high blockage ribs channel. Experimental Thermal and Fluid Science 83 (2017) 248-259.

26. Cengel Y, Cimbala JM. Fluid mechanics fundamentals and applications. International Edition, McGraw Hill Publication, 185201,2006

27. Goktepeli I, Atmaca U, Cakan A. Investigation of heat transfer augmentation between the ribbed plates via Taguchi approach and Computational Fluid Dynamics. Journal of Thermal Science 29 (2020) 647-666

28. Matsubara K, Ohta H, Miura T. Entrance region heat transfer in a channel with a ribbed wall. Journal of Heat Transfer 138 (2016) 122001.

29. Anonymous. ANSYS-Fluent 12.0 Theory Guide. ANSYS Inc (2009)

30. Goktepeli I, Atmaca U, Yagmur S. Visualization of flow characteristics between the ribbed plates via Particle Image Velocimetry. Thermal Science 25 (2021) 171-179.

31. Yemenici O, Umur H. Experimental aspects of heat transfer enhancement over various flow surfaces. Heat Transfer Engineering 37 (2016) 435-442. 